\title{
Innate immunity mediated by TLR9 modulates pathogenicity in an animal model of multiple sclerosis
}

\author{
Marco Prinz, ${ }^{1}$ Folker Garbe, ${ }^{1}$ Hauke Schmidt, ${ }^{1}$ Alexander Mildner, ${ }^{1}$ Ilona Gutcher, ${ }^{2}$ Karina Wolter, \\ Matthias Piesche, ${ }^{3}$ Roland Schroers, ${ }^{3}$ Elisabeth Weiss, ${ }^{4}$ Carsten J. Kirschning, ${ }^{5}$ \\ Christian D.P. Rochford, ${ }^{6}$ Wolfgang Brück, ${ }^{1}$ and Burkhard Becher ${ }^{2}$

\begin{abstract}
${ }^{1}$ Institute of Neuropathology, Georg-August-University, Gottingen, Germany. ${ }^{2}$ Department of Neurology/Neuroimmunology Unit, Universitätsspital, University of Zurich, Zurich, Switzerland. ${ }^{3}$ Department of Hematology and Oncology and ${ }^{4}$ Department of Radiation Oncology, Georg-August University, Gottingen, Germany. Institute of Medical Microbiology, Immunology and Hygiene, Technical University of Munich, Munich, Germany. ${ }^{6}$ Neuroimmunology Unit, European Neuroscience Institute, Gottingen, Germany.
\end{abstract}

\begin{abstract}
Inflammatory diseases of the CNS, such as MS and its animal model EAE, are characterized by infiltration of activated lymphocytes and phagocytes into the CNS. Within the CNS, activation of resident cells initiates an inflammatory cascade, leading to tissue destruction, demyelination, and neurologic deficit. TLRs recognize microbes and are pivotal mediators of innate immunity. Within the CNS, augmented TLR expression during EAE is observed, even in the absence of any apparent microbial involvement. To determine the functional relevance of this phenomenon during sterile autoimmunity, we studied the role of different TLRs as well as their common signaling adaptor MyD88 in the development of EAE. We found that $\mathrm{MyD88}^{-/-}$mice were completely EAE resistant. Surprisingly, this protection is partly due to engagement of the CpG receptor TLR9. Restricting the MyD88 or TLR9 mutation to host radio-resistant cells, including the cells within the CNS, revealed that engagement of radio-resistant cells modulated the disease course and histopathological changes. Our data clearly demonstrate that both TLR9 and MyD88 are essential modulators of the autoimmune process during the effector phase of disease and suggest that endogenous "danger signals" modulate the disease pathogenesis.
\end{abstract}

\section{Introduction}

MS is considered to be an inflammatory demyelinating disease of the CNS, the etiology of which remains unclear (1). It is generally held that cell-mediated immunity underlies the pathogenesis, based on the identification of inflammatory infiltrates in MS lesions of CNS white matter. This condition is represented in the developed animal model for brain inflammation and MS, known as EAE, which is a vital tool to study the neuroimmunological events occurring during the disease (2). In both EAE and MS, there are 2 main phases that constitute the sequence of immunopathological events: an initial priming/activation phase in which autoaggressive lymphocytes are activated and a subsequent recruitment and effector phase in which such cells invade the CNS and cause tissue destruction. For efficient $\mathrm{T}$ cell priming in secondary lymphoid tissues as well as for the maintenance of an encephalitogenic immune response within the CNS, competent APCs such as dendritic cells are an absolute requirement (3). Activation and maturation of these APCs is generally mediated through the presence of a so-called danger signal $(4,5)$. These signals, derived from microbes or damaged tissue, are required to break immunological unresponsiveness or tolerance for the development of an autoimmune response (6). Host organisms detect "danger" by recognizing pathogen-associated molecular patterns or stress-induced self molecules (7). TLRs are crucial mediators of such responses. Toll is a key receptor in antifungal immunity

Nonstandard abbreviations used: Ag, antigen; APP, amyloid precursor protein; dpi, days after immunization; KLH, keyhole limpet hemocyanin; MOG, myelin oligodendrocyte glycoprotein; PTX, pertussis toxin.

Conflict of interest: The authors have declared that no conflict of interest exists. Citation for this article: J. Clin. Invest. 116:456-464 (2006). doi:10.1172/JCI26078. of Drosophila (8). TLRs are mammalian Toll homologues expressed mostly by immune cells. At least 11 members of the TLR family exist. At present, ligands have been identified for TLR1 through TLR9. Most of these ligands are derived from pathogens and comprise a very heterogeneous group, including viruses, bacteria, DNA, RNA, toxins, chemical compounds, and others.

MyD88 is a common cytoplasmic adapter protein, which associates as an obligate functional partner with most members of the TLR and IL-1 receptor family (9-11). Therefore, $M y D 88^{-/-}$mice do not show any response to IL-1 family cytokines in vivo (12), are resistant to LPS-induced endotoxin shock syndrome (13), and fail to respond to peptidoglycans, lipoproteins (14), antiviral compounds (15), and CpG DNA (16) as well as flagellins (17).

To induce autoimmune diseases such as EAE, putative TLR ligands such as $\mathrm{CPG}$ are commonly used to break peripheral tolerance and to induce a pathogenic response against the CNS (18). In addition to the requirement for TLR signaling to induce immunity, CNS-resident microglia have been shown to express an array of different TLRs, depending on their state of activation (19-21). Yet, their in vivo role and function in the context of a sterile immune attack against the CNS remain to be established.

Here we demonstrate that MyD88 expression by radio-resistant cells, such as brain endogenous cells, is vital for their capacity to drive disease development. This is at least partly due to the action of the $\mathrm{CPG}$ receptor TLR9 even though $\mathrm{CPG}$ is not detectable during the effector phase of EAE within the CNS. Our findings provide definitive in vivo evidence for the involvement of brainrestricted TLRs during sterile inflammation, and we propose that endogenous TLR ligands may act as maturation stimuli for CNSresident cells during disease pathogenesis. 


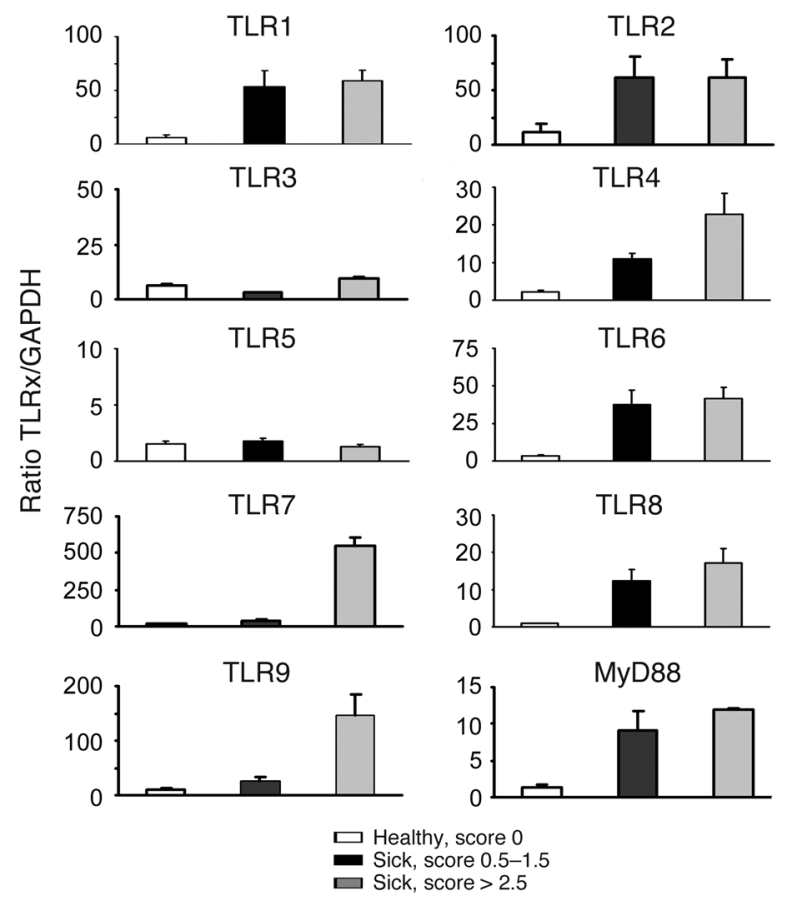

\section{Results}

Induction of TLR expression within the brain in a model of CNS-specific autoimmunity. Previously, upregulation of TLR expression by CNS resident cells, in particular by microglia, in response to different inflammatory stimuli was reported in vitro $(19,21)$. To assess whether a sterile inflammatory response can mediate TLR regulation in vivo, we examined the CNS for TLR mRNA expression by real-time PCR. Spinal cords from 3 different time points were evaluated for the expression of TLR1, TLR2, TLR3, TLR4, TLR5, TLR6, TLR7, TLR8, TLR9, and MyD88. These time points were as follows: (a) preclinical, no animals exhibiting disease symptoms (score 0); (b) disease onset, animals showing first symptoms of EAE (score 0.5-1.5); and (c) peak EAE (score $\geq 2.5$ ). We detected a remarkable increase of TLR1, TLR2, TLR4, TLR6, TLR7, TLR8, and TLR9 mRNA during the progression of disease (Figure 1). TLR3 and TLR5 mRNA expression levels were lower than TLR1, TLR2, TLR4, TLR6, TLR7, and TLR9 levels and remained unchanged during the course of disease. TLR1, TLR2, TLR4, TLR6, TLR8, and MyD88 expression peaked at the first clinical signs of disease, coinciding with leukocyte influx. In contrast, levels of TLR7 and TLR9 mRNA increased dramatically during the peak of disease, suggesting a response by CNS resident cells during the effector phase of disease. Taken together, these data demonstrate that the expression of both TLRs and their main signaling molecule, MyD88, is modulated during EAE development.

The role of TLR signaling during the priming phase of EAE. To judge to what extent the absence of TLRs and its signaling molecule MyD88 affects the course of a sterile autoimmune CNS disease, EAE was induced in mice deficient for TLR2, TLR9, and MyD88. After immunization with myelin oligodendrocyte glycoprotein (MOG) peptide emulsified in CFA, all WT and TLR2 $2^{--}$mice developed EAE with an incidence of $100 \%$ and a similar mean disease onset and severity (Figure 2A and Table 1). These results clearly demonstrate that TLR2 is dispensable for the induction and progression of disease. In contrast, $M y D 88^{-/-}$mice were completely EAE resistant, and

\section{Figure 1}

TLR regulation during autoimmune CNS disease. Expression of TLRs and MyD88 in the CNS of EAE-diseased mice. TLR1-9 and MyD88 mRNA were detected in spinal cord tissue samples by using real-time PCR at different stages of disease. Immunized, nonsick animals (score 0 ) are indicated in white, slightly diseased mice (score $0.5-1.5$ ) in black, and mice with severe EAE (score > 2.5) in gray. Data are expressed as ratio of induced TLR to endogenous GAPDH and expressed as SEM.

TLR9-/- mice developed EAE with a significant delay in disease onset (17.9 days after immunization [dpi]) and weak clinical manifestations (mean clinical score $=1.9$ ) compared with WT mice (mean day of disease onset $=15.9 \mathrm{dpi}$; mean maximal clinical score $=2.5$ ). The data indicate that both TLR9 and MyD88 are needed for EAE induction and progression. The fact that TLR9-/- mice demonstrate some degree of EAE resistance could potentially be explained by the fact that the adjuvant used to induce immunity (heat-killed Mycobacterium tuberculosis H37RA) engages the TLR9 receptor. In order to determine the interaction between $M$. tuberculosis and TLR9, we investigated the mechanisms of cellular activation using an NF-кB reporter assay (Figure 2B). Exposure of transgenic TLR9-bearing HEK293 cells to mycobacterial H37RA did not result in NF-KBdriven reporter gene activation. The concentrations used correlated to those used to immunize mice. The lack of TLR9-related activation indicates the absence of detectable DNA containing unmethylated (CpG) sequences in the H37RA preparation. However, the same amount of mycobacterial material was clearly sufficient to induce activation of 1 of its natural receptors, TLR2 (data not shown). Similar results with H37RA were obtained when murine instead of human TLR9 was introduced in HEK293 cells (data not shown). Moreover, neither MOG peptide nor pertussis toxin (PTX) induced a measurable activation of TLR9. Therefore, the decreased EAE susceptibility of TLR9-/- mice to EAE is most likely not mediated by the interaction of adjuvant or antigen (Ag) with TLR9, but rather implicates an endogenous TLR9-dependent signal to exert an encephalitogenic effect.

To determine the capacity of these mice to generate a primary immune Ag-driven response, WT, TLR2-/-, TLR9-/-, and $M y D 88^{-/-}$ mice were immunized with either $\mathrm{MOG}_{35-55}$ peptide or keyhole limpet hemocyanin (KLH) emulsified in CFA. Seven days later, LNs were removed, and the capacity of lymphocytes to respond to their cognate Ag was measured in a recall assay. Figure 2, C and D, shows that lymphocytes derived from WT, TLR2- and TLR9-deficient mice developed comparable proliferative responses and produced similar levels of IFN- $\gamma$. T cell proliferation in TLR9-/- mice was

\section{Table 1}

Requirement of normal TLR signaling for active EAE in mice

$\begin{array}{lccc}\begin{array}{l}\text { Mouse } \\ \text { genotypes }\end{array} & \begin{array}{c}\text { Incidence } \\ (\%)\end{array} & \begin{array}{c}\text { Mean day of } \\ \text { disease onset }^{A}\end{array} & \begin{array}{c}\text { Mean maximal } \\ \text { clinical score }( \pm \text { SEM })^{A}\end{array} \\ \text { WT } & 8 / 8(100) & 15.9 & 2.5 \pm 0.3 \\ \text { TLR2 }{ }^{-\alpha} & 7 / 7(100) & 14.5 & 2.6 \pm 0.3 \\ \text { TLR }{ }^{-\alpha} & 8 / 9(89) & 17.9 & 1.9 \pm 0.2 \\ \text { MyD88- } & 0 / 6(0) & \text { n.d. } & \text { n.d. }\end{array}$

Shown is one representative experiment of 3 independent experiments presented in Figure 2A. Table summarizes data shown in Figure 5. ADiseased mice. n.d., no disease. 

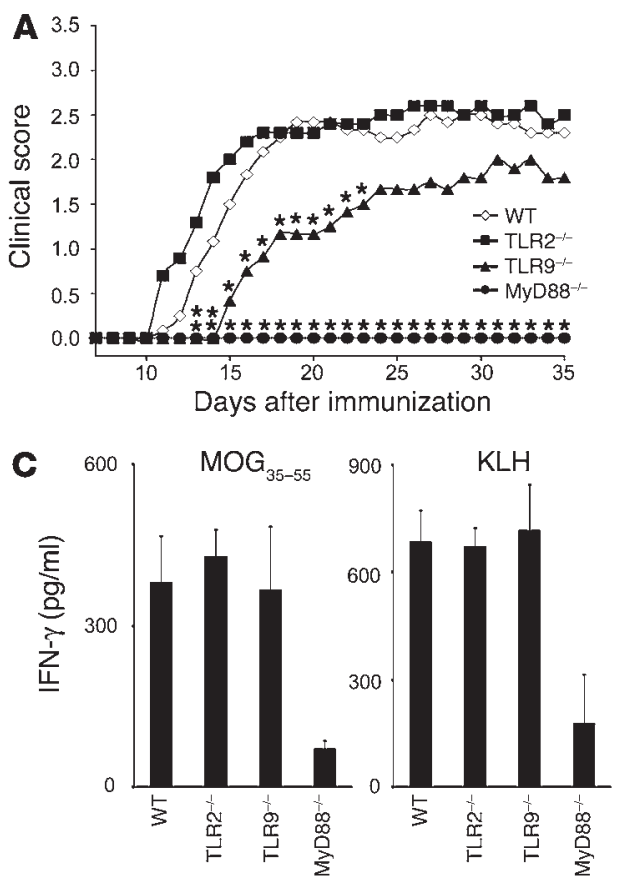
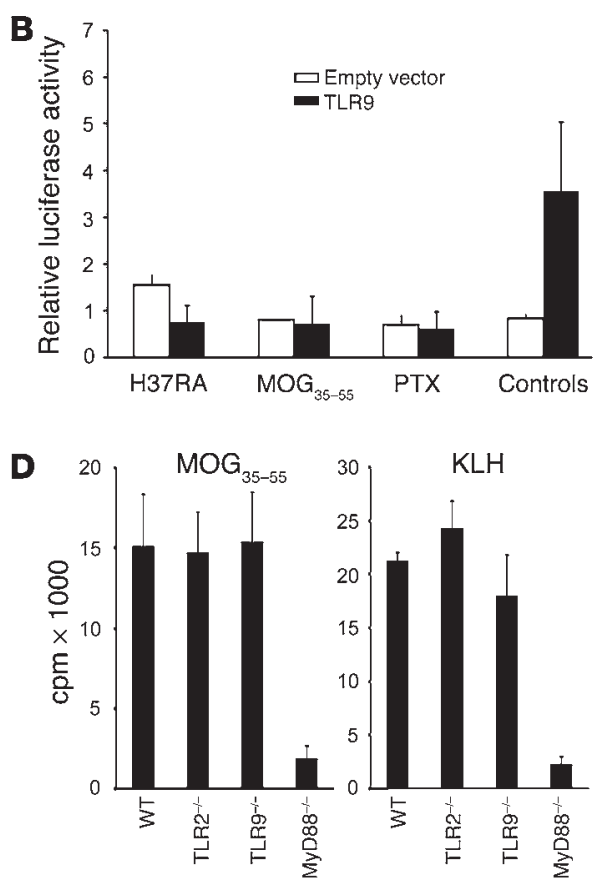

Figure 2

TLR signaling is critical for the induction of active MOG-EAE in C57BL/6 mice and the diseasespecific Th1 response. (A) EAE was induced by active immunization of WT (diamonds), TLR2 $2^{-/}$ (squares), TLR9-/- (triangles), and MyD88-deficient (circles) mice. Each data point represents the mean of 6 or more animals. Statistically significant data points are marked with asterisks $(P<0.05)$. One representative set of experiments out of 3 is shown. (B) TLR9 engagement by selective EAE immunization components in vitro. Human TLR9 was ectopically expressed in HEK293 cells. Cells were stimulated with either $5 \mu \mathrm{l} M$. tuberculosis cell wall components (H37RA, $50 \mu \mathrm{g} / \mathrm{ml}$ ), $20 \mu \mathrm{l}$ of $\mathrm{MOG}_{35-55}$ peptide $(4 \mu \mathrm{g} / \mu \mathrm{l})$ in sterile PBS, $20 \mu \mathrm{l} \mathrm{PTX}(1.25 \mathrm{ng} / \mu \mathrm{l})$ in endotoxin-free water, or TLR-specific stimuli ( $2 \mu \mathrm{M}$ oligodeoxynucleotide 2006). Luciferase activity in cellular lysates was measured, normalized to $\beta$-galactosidase activity, and related to activity in unstimulated cells in each group of vector/TLR transfectants. The empty vector is indicated by white bars and TLR 9 by black. The results are representative of more than 3 independent experiments. (C and D) $M y D 88^{-/-}$but not $T L R 9^{-/-}$mice failed to generate Th1 immune responses. Recall responses of $L N$ cells to either $M \mathrm{G}_{35-55}$ peptide or $\mathrm{KLH}$ were measured by IFN- $\gamma$ ELISA (C) and [H]thymidine-uptake (D). Data shown are representative of at least 2 independent experiments.

always similar to WT lymphocytes, even when other Ag concentrations were used (data not shown). In contrast, $M y D 88^{-/-}$mice did not develop measurable primary responses to their cognate $\mathrm{Ag}$, as assessed by the recall assay. It has been suggested that $\mathrm{CD} 4{ }^{+} \mathrm{CD} 25^{+}$ Tregs could potentially account for the insufficient $\mathrm{T}$ cell priming in $M y D 88^{-/-}$mice (22). To assess the role of Tregs in MyD88-dependent T cell priming, WT and MyD88-deficient mice were intravenously injected with $100 \mu \mathrm{g}$ of monoclonal anti-CD25 antibody or the respective isotype control 3 days before immunization. This treatment led to a depletion of Tregs, as shown in Supplemental Figure 1A (supplemental material available online with this article; doi:10.1172/JCI26078DS1). Subsequent measurement of T cell proliferation upon stimulation by the cognate Ag in vitro revealed an enhanced proliferation in WT lymphocytes whereas $M y D 88^{-1}$ lymphocytes were still not able to expand properly (Supplemental Figure $1 \mathrm{~B}$ ). Therefore, the inability to induce autoimmunity in $M y D 88^{-/-}$mice can be explained by their poor T cell priming, independent of $\mathrm{CD}^{+} \mathrm{CD} 25^{+}$Treg cells. However, TLR9-/- mice show normal $\mathrm{T}$ cell priming, and the decreased disease course cannot solely be explained by inefficient priming/expansion.
Altered CNS pathology in the absence of TLR signaling. In order to determine both the extent and the composition of CNS-infiltrating cells and subsequent pathological changes over time, spinal cords were examined histologically at early (20 dpi; Figure 3A) and late (35 dpi; Figure 3B and Supplemental Table 1) disease time points. Figure 3A and Supplemental Table 1 show a plethora of infiltrating macrophages in WT and TLR9-deficient mice during the early phase of clinical EAE. This influx was accompanied by the invasion of CD3-positive lymphocytes and amyloid precursor protein-positive (APP-positive) axonal structures, indicating comparable acute axonal damage in these animals. Accordingly, the demyelinated area was similar in these genotypes during the early phase (Supplemental Table 1). However, $M y D 88^{-/-}$mice displayed no apparent inflammation. These distinct infiltration features reflect the clinical behavior of this line with high resistance against EAE. Spinal cord sections from mice displaying the most severe symptoms at day 35 stained for Mac-3-positive macrophages/microglia revealed similar perivascular and meningeal distribution of the infiltrates in inflamed spinal cords of both WT and TLR2-/mice (Figure 3B). Quantification of histopathological manifestations on spinal cord sections showed similar numbers of invading macrophages in WT and TLR2 ${ }^{-/-}$mice (Supplemental Table 1). Accordingly, the number of CD3-positive cells was also similar in both genotypes. Moreover, the myelin damage expressed as the percentage of demyelinated area as of total white matter was compatible in TLR2-/- and WT mice. Surprisingly, however, TLR9-deficient mice revealed in the chronic phase much smaller infiltrating foci in the spinal cord of diseased mice (Figure 3B, third line) with fewer invading hematopoietic cells compared with WT mice. APP-stained deposits did not increase when compared with the histology obtained at disease onset, and the extent of the demyelinated area $(1.8 \% \pm 0.4 \%)$ was strongly reduced in TLR9-deficient mice. As expected, EAE-resistant $M y D 88^{-/-}$mice were virtually free of any leukocytic infiltrates and axonal changes (Figure 3B). Overall, these data indicate that the absence of MyD88 and TLR9, but not of TLR2, significantly alters the degree and composition of inflammation, demyelination, and axonal damage during late disease phase.

TLR engagement on CNS resident cells determines the course of EAE. In order to determine whether TLR engagement of hematopoietic or stromal cells such as CNS resident cells contributes to the development and progression of EAE, BM chimeric mice were generated in which either TLR9 or MyD88 expression was limited to the 
A

Mac-3

CD3

LFB

APP
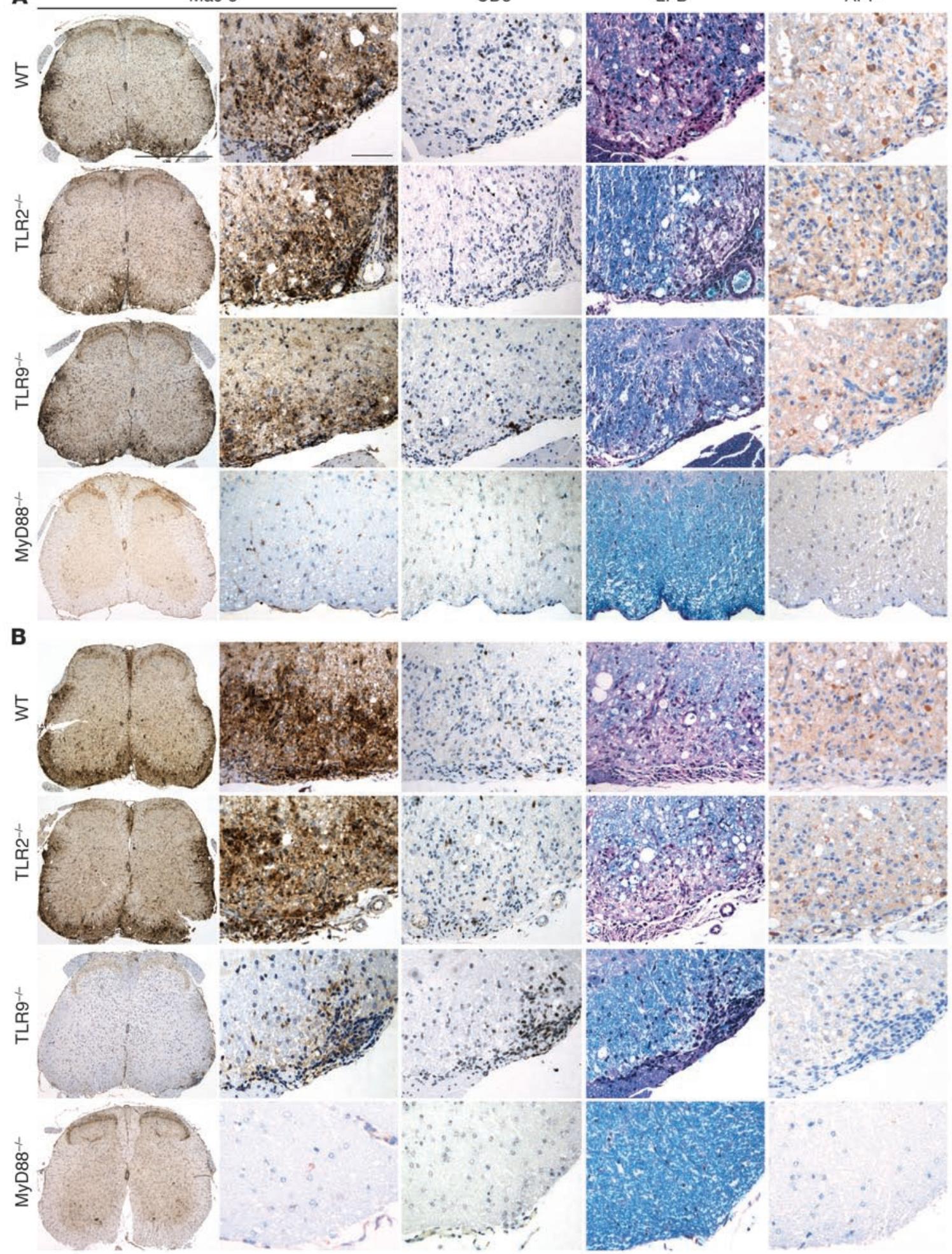

Figure 3

Diminished CNS inflammation and axonal damage in MyD88- and TLR9-deficient mice. (A and B) Immunohistochemistry from WT and $T L R 2^{-/-}, T L R 9^{-/-}$, and $M y D 88^{-/-}$mice. In all cases, animals with the highest clinical signs were taken either at peak of disease (day 20, A) or at the end of the experiment (day 35, B). Mac-3 staining in total spinal cord sections revealed similar strong macrophage infiltration in WT, $T L R 2^{-/-}$, and $T L R 9^{-/-}$mice at day 20 (A) whereas at later time points, $T L R 9^{-/-}$mice revealed smaller infiltrates and $M y D 88^{-/-}$mice revealed no infiltrates (B). Higher magnifications show CD3-positive lymphocytes, macrophages (Mac-3), regions of demyelination (luxol fast blue, LFB), and APP deposits representing axonal damage (APP). Scale bars: $500 \mu \mathrm{m}$ (first column); $30 \mu \mathrm{m}$ (second column). 
A

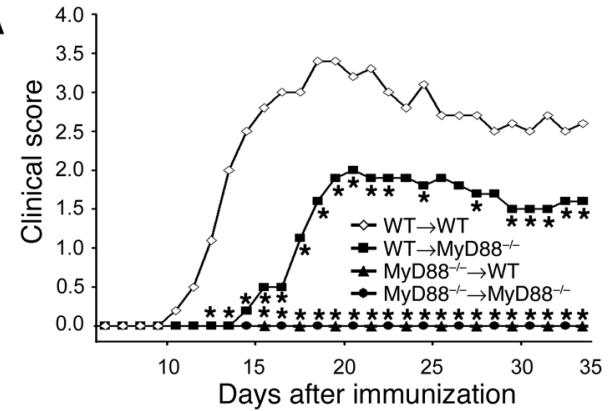

B

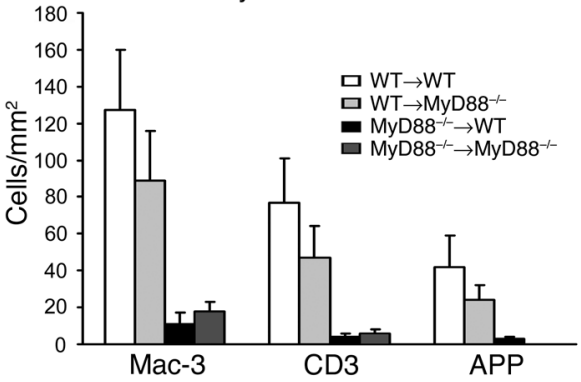

C

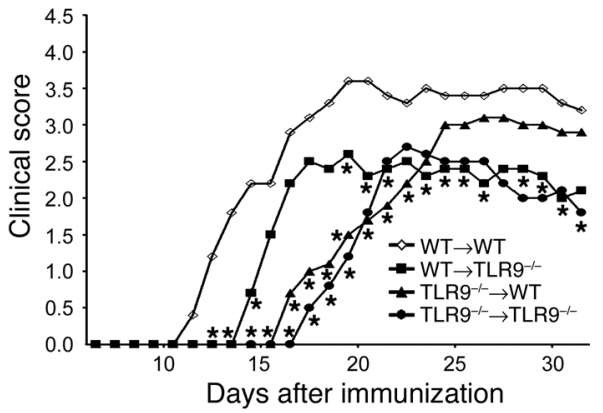

radio-resistant or radio-sensitive compartment of the host. To do so, we combined BM chimerism with gene targeting as described earlier (23-26). Two months after reconstitution of congenic WT mice $(\mathrm{CD} 45.1=\mathrm{Ly} 5.1)$ with $M y D 88^{-/-} \mathrm{BM}(\mathrm{CD} 45.2=$ Ly5.2), surface staining of peripheral blood revealed that the donor marker CD45.2 was detectable on virtually all blood cells (Supplemental Figure 2A). More detailed quantitative blood analysis of the BM chimeric mice revealed a virtually complete reconstitution of $\mathrm{B}$ lymphocytes and macrophages, whereas $\mathrm{T}$ lymphocyte engraftment was slightly less effective, as described earlier (25) (Supplemental Figure 2 and data not shown). In contrast, CNS-resident cells displayed the host genotype and were not significantly replaced by BM elements (Supplemental Figure 2C). Our findings match the reports by others concerning the limited reconstitution of the CNS by hematopoietic myeloid cells after lethal irradiation (27-29). To assess functional reconstitution of the lymphoreticular compartment, systemic immune responses were evaluated in MyD88 BM chimeric mice (Supplemental Figure 2B). Chimeras were immunized with $\mathrm{MOG}_{35-55}$ in CFA, and $\mathrm{LN}$ cells were isolated at $7 \mathrm{dpi}$ for the assessment of MOG recall responses in

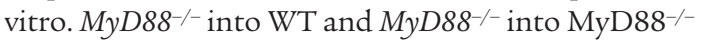
chimeras did not generate strong $\mathrm{T}$ cell responses to MOG, as evaluated by IFN- $\gamma$ production and proliferation. In contrast, both WT into $\mathrm{MyD} 88^{-/-}$and WT into WT mice demonstrated equivalent capacities to

Table 2

\section{Figure 4}

Nonhematopoietic TLR signaling is involved in EAE disease. (A-C) Active EAE in MyD88 (A and B) and TLR9 (C) BM chimeric mice. After $\mathrm{BM}$ reconstitution, mice were allowed to recover for 6-8 weeks, then immunized with $\mathrm{MOG}_{35-55}$ in CFA as described and scored for disease (means). Statistically significant data points are marked with asterisks. $P<0.05$. Results are representative of 2 independent experiments. (B) Quantification of mononuclear infiltrates and axonal damage in MyD88 chimeric mice. For each genotype, at least 4 diseased spinal cords were used for quantification (means $\pm S D$ ). Arrows indicate direction of bone marrow reconstitution.

prime $\mathrm{T}$ cells and for those $\mathrm{T}$ cells to proliferate and secrete IFN- $\gamma$ when challenged with their cognate Ag in vitro. We can conclude that MyD88 expression on hematopoietic cells is essential for the priming of encephalitogenic T cells. However, the lack of MyD88 expression on radio-resistant nonhematopoietic cells in the WT into $M y D 88^{-/-}$chimeras appears to have no impact on the peripheral priming and differentiation of Ag-reactive T cells. Accordingly, chimeras immunized with MOG peptide in CFA were protected from $\mathrm{EAE}$ when the donor $\mathrm{BM}$ was of the $\mathrm{MyD} 88^{-/}$genotype (Figure $4 \mathrm{~A}$ and Table 2). The lack of MyD88 from the radio-resistant compartment, e.g., the CNS parenchyma (WT into MyD88-/-), caused a significant delay in disease onset and reduced clinical severity compared with chimeric mice that expressed MyD88 on stromal cells (WT into WT). In addition, detailed immunohistological analysis of immunized chimeras revealed a distinct lesion pattern (Figure 4B and Supplemental Figure 3). The number of infiltrating Mac- $3^{+}$macrophages and $\mathrm{CD}^{+}$lymphocytes was strongly decreased when MyD88 was absent from the nonhematopoietic compartment. This decrease of mononuclear infiltrates correlated with fewer APP deposits, thus indicating that the presence of MyD88 also regulates axonal damage during brain disease. To assess the contribution of TLR9 to the MyD88-mediated radioresistant nonhematopoietic effects, BM chimeras were generated using TLR9-/- mice as BM recipients (Figure 4C). As shown with unmanipulated TLR9 $9^{-1}$ mice, the absence of TLR9 on the hematopoietic compartment in chimeras strongly delayed disease onset (TLR9--- into WT and TLR9-/-into TLR9--). Intriguingly, there was also an obvious effect when TLR9 was specifically absent from the radio-resistant compartment alone (WT into TLR9-/- compared with WT into WT chimeras). Accordingly, quantification of histopathological changes within the CNS, including infiltration

EAE in MyD88 and TLR9 BM chimeric mice

\begin{tabular}{|c|c|c|c|c|}
\hline $\begin{array}{l}\text { Genotype } \\
\text { BM donor }\end{array}$ & $\begin{array}{l}\text { Genotype } \\
\text { BM recipient }\end{array}$ & $\begin{array}{c}\text { Incidence } \\
(\%)\end{array}$ & $\begin{array}{l}\text { Mean day of } \\
\text { disease onset }{ }^{A}\end{array}$ & $\begin{array}{c}\text { Mean maximal } \\
\text { clinical score }( \pm \text { SEM })^{A}\end{array}$ \\
\hline WT & WT & $8 / 8(100)$ & 14.7 & $3.4 \pm 0.3$ \\
\hline WT & MyD88-/- & 7/8 (88) & 18.2 & $2.1 \pm 0.2$ \\
\hline MyD88-/- & WT & $0 / 7(0)$ & n.d. & n.d. \\
\hline MyD88-/- & MyD88 ${ }^{-/}$ & $0 / 6(0)$ & n.d. & n.d. \\
\hline WT & WT & $6 / 6(100)$ & 15.4 & $3.6 \pm 0.4$ \\
\hline WT & TLR9-/- & $5 / 5(100)$ & 17.4 & $2.3 \pm 0.3$ \\
\hline TLR9-/- & WT & $9 / 10(90)$ & 20.8 & $2.8 \pm 0.3$ \\
\hline TLR9-/- & TLR9-/- & 7/8 (88) & 21.4 & $2.4 \pm 0.2$ \\
\hline
\end{tabular}

Table shows clinical parameter of MOG-induced EAE in BM chimeric MyD88-/- and

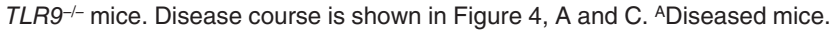




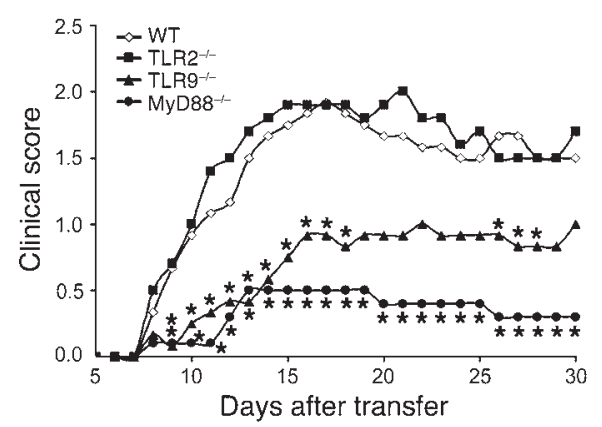

Figure 5

Encephalitogenic $T$ cells are less effective at inducing EAE when transferred into MyD88- or TLR9-deficient hosts. Adoptive transfer of MOGreactive T cells $\left(25 \times 10^{6}\right.$ cells $)$ into $M y D 88^{-/-}$or $T L R 9^{-/-}$but not $T L R 2^{-/-}$ hosts results in milder disease. Data shown are from 1 representative experiment of at least 2 individual experiments with at least 5 mice per group (means). Asterisks indicate statistical significance. $P<0.05$.

by macrophages and $\mathrm{T}$ cells as well as axonal and myelin damage, revealed reduced alterations when TLR9 was absent from the radio-resistant compartment (Supplemental Table 3). In addition, $\mathrm{T}$ cell priming was always normal in TLR9 BM chimeras (data not shown). Hence, the lack of TLR9 from the radio-resistant cells, such as CNS parenchyma, endothelial, or stromal cells, accounts for a significant portion of the MyD88-mediated effects in the nonhematopoietic compartment.

The role of TLR signaling during the effector phase of EAE. Both $M y D 88^{-1-}$ and TLR9-1- mice displayed resistance to developing EAE upon active immunization with MOG peptide and CFA, including putative TLR ligands. As we were not able to detect an overt deficiency in T cell priming in TLR9 ${ }^{-/-}$mice, we wanted to assess the role and function of TLR9 and its adaptor molecule MyD88 during the effector phase of EAE in the absence of any microbial components commonly used as adjuvant. In order to do so, encephalitogenic MOG-reactive lymphocytes from WT mice were generated, restimulated in vitro, and adoptively transferred into either WT, $M y D 88^{-/}$, or TLR9-/- mice. Figure 5 shows that $\mathrm{MyDS8}^{-/-}$and TLR9-/- mice developed EAE with a significantly later disease onset and severity. The data indicate that both MyD88 and TLR9 are involved in the local maintenance of encephalitogenicity during the effector phase of EAE within the CNS. TLR2-deficient mice, however, had a similar course of disease compared with WT recipients, emphasizing the redundant role of TLR2 for EAE pathogenesis (Table 3). Taken together, our data clearly demonstrate that specific members of the TLR family and its signaling molecule MyD88 that were previously thought to be involved only in germ-specific host defense are also crucial elements for the generation of encephalitogenic $T$ cells in the priming phase as well as for the maintenance of encephalitogenicity during the sterile effector phase of EAE.

\section{Discussion}

Environmental factors strongly influence the development of autoimmune disease, including MS (30-32), and elements of both the adaptive and innate immune systems are transcribed and expressed in MS lesions (33). Components of the innate immune system are involved in several deleterious steps in the autoimmune cascade, including activation of myelin-reactive $\mathrm{T}$ lymphocytes by APCs and the development of membrane attack complexes in the CNS of MS patients. Despite the generally accepted view that activation of innate immunity by specific TLR ligands, such as bacterial cell wall components and DNA, are required for the generation of a productive immune response, the role of CNSspecific innate immunity in this process is poorly understood. While the adjuvant used to elicit peripheral immunity contains mycobacterial extracts to act as "danger signals," the adjuvant is localized subcutaneously to stimulate local APCs that subsequently migrate into the regional LNs. After successful T cell priming, autoaggressive lymphocytes subsequently migrate into the CNS to cause tissue damage. Within the CNS, no microbial products are available to drive innate immune activation of CNS residents. As we have shown recently, the activation of CNS resident cells is vital for the development and maintenance of inflammatory CNS lesions $(23,24,34)$. However, whether the innate immune system also participates in the conditioning of the CNS remains elusive. The goal of our study was to determine the functional relevance of innate immunity within the CNS in vivo. Here we provide definitive evidence that a sterile inflammatory response within the CNS requires innate immune activation of CNS-resident cells. Initially, we found that during EAE, TLR expression is strongly increased within the CNS. We did not distinguish between TLR expression by invading leukocytes or CNS-resident neuronal or glial elements. However, microglia acting as CNS sentinels for danger signals have been shown to increase TLR expression in response to inflammatory mediators as well as microbial products (19). To determine whether the modulation of the TLR profile has any functional significance to the development of EAE, mice lacking specific TLRs were immunized against MOG peptide. Interestingly, TLR2 $2^{-/}$mice were fully EAE susceptible, even when the adjuvant used contained mycobacterial extracts, which are thought to elicit cell activation mainly through TLR2. However, in accordance with our findings, others have suggested TLR2-independent pathways for mycobacterial components (35). Both MyD88 and TLR9 were found to be crucial for the formation and maintenance of inflammatory lesions. We found that mice deficient in MyD88 were not able to elicit a productive immune response. Hence, the EAE resistance of MyD88-deficient mice is probably largely due to the requirement of MyD88 in T cell priming. Moreover, depletion of regulatory $\mathrm{T}$ cells could, in our hands, only enhance $\mathrm{T}$ cell proliferation in WT mice but not restore $\mathrm{T}$ cell priming deficiency in $M y D 88^{-/-}$mice. Thus, mechanisms of $\mathrm{T}$ cell priming in $M y D 88$-deficient mice to MOG peptide might be different from Ags used in previous studies $(22,36)$. To assess the functional significance of TLR expression within the CNS, we used BM chimeras in which TLR9 and MyD88 were deleted specifically from the radio-resistant nonhematopoietic compartment, e.g., brain, spinal cord, or endothelial cells. The

\section{Table 3}

Adoptive transfer of MOG-reactive lymphocytes into WT and TLR-deficient recipients

\begin{tabular}{|c|c|c|c|}
\hline $\begin{array}{l}\text { Mouse } \\
\text { genotypes }\end{array}$ & $\begin{array}{c}\text { Incidence } \\
(\%)\end{array}$ & $\begin{array}{c}\text { Mean day of } \\
\text { disease onset }{ }^{A}\end{array}$ & $\begin{array}{c}\text { Mean maximal } \\
\text { clinical score }( \pm \text { SEM })^{A}\end{array}$ \\
\hline WT & $6 / 6(100)$ & 8.2 & $2.0 \pm 0.4$ \\
\hline$T L R 2^{-/-}$ & $5 / 5(100)$ & 8.6 & $2.1 \pm 0.3$ \\
\hline$T L R 9^{-/-}$ & $5 / 6(83)$ & 11.8 & $1.4 \pm 0.3$ \\
\hline MyD88-/- & $3 / 5(60)$ & 11.0 & $0.8 \pm 0.3$ \\
\hline
\end{tabular}

Table summarizes data shown in Figure 5. ADiseased mice. 
results obtained with $M y D 88$-deficient mice point to an essential role of TLR signaling and/or IL-18/IL-1 $\beta$ in the pathogenesis of EAE. However, it is unlikely that signaling through members of the IL-1 receptor family alone is responsible for the relative resistance of the mice to EAE. The EAE resistance of mice in which MyD88 is absent from nonhematopoietic cells is at least in part due to the actions of the TLR9 receptor. Mice in which TLR9 was deleted from the radio-resistant compartment, such as CNS, developed EAE with delayed kinetics and severity. Previously, TLR9 has been identified as the key receptor in the recognition of $\mathrm{CPG}$ motifs (37). However, despite the fact that we were not able to detect any CPGs in our mycobacterial preparation, TLR9 was found to be crucial for the course of EAE. So far we cannot formally exclude the possibility that our in vitro HEK293 system lacks additional coreceptors needed for TLR9 signaling or that CpGs are already degraded in the mycobacteria preparation. Our results from challenge of fibroblasts that ectopically overexpress TLR9 or TLR9-/LN cells with the compounds used for EAE induction clearly indicate that it is not the initial challenge with $\mathrm{MOG}_{35-55}$ peptide, $\mathrm{PTX}$, or mycobacterial lypophilisates, but rather secondary effects, that elicited a TLR9-dependent signal. Accordingly, adoptive transfer of encephalitogenic $\mathrm{T}$ cells into TLR9-/- recipients revealed that TLR9 engagement drives autoimmunity even under completely sterile conditions in the absence of microbial products. This finding implicates additional endogenous, disease-associated ligands apart from CpGs. Indeed, as we have recently shown, phosphodiester DNA is able to stimulate TLR9 independently of DNA sequence specificity or methylation status, especially if the DNA has been transferred to the endosome (38). Therefore, it is tempting to speculate that TLR9 activation in vivo can be achieved by any kind of DNA independent of its origin but correlating with its amount. Potential candidates in the EAE model include, for example, DNA motifs released by apoptotic inflammatory $T$ cells during EAE (39). In addition, TLR9 was found to be essential for cellular activation by IgG2a autoantibodies together with self DNA in the absence of bacterial CpGs in a mouse model of autoimmune systemic disorder (40). The authors claimed that during autoimmune disease the adaptive immune response is sustained by the adjuvant effect of endogenous nucleic acids. This leaves open the question of what is primary, but without doubt, host DNA exhibits innate immunostimulatory potential, $\mathrm{CpG}$ methylation notwithstanding. In addition, other TLRs might also be involved. For example, Kerfoot and colleagues have shown that TLR4 deficiency somewhat modulates disease activity and have claimed that the PTX used to induce EAE mediates its effects partially through TLR4 (41). Therefore, MyD88-dependent effects in our model might also be, at least in part, due to engagement of TLR4.

Activation of CNS residents facilitates the formation of inflammatory lesions within the CNS. The most important population of CNS residents to interact with invading immune cells are microglia. The fact that microglia carry a host of TLRs has been described elsewhere, but the significance of this finding has been related only to bacterial infections of the brain. Over the past few years, we have learned that innate immune activation is required for the development of a productive adaptive immune response. Yet, under sterile conditions, the same parameters that govern antimicrobial immunity are also thought to underlie sterile immune responses (6). Beutler proposed the novel concept of "innate autoimmunity," combining the assumption that aberrant TLR signaling without microbes over a sustained period of time concomitant with Ag presentation might result in an adaptive immune response to host molecules with the observation that some endogenous molecules signaling through TLRs likely do so because sterile inflammation so closely resembles infectious inflammation. Our findings strongly support this notion, and it appears that the CNS itself harbors the innate capacity to orchestrate a sterile immune response within its realm. The tantalizing task remaining is to discover which endogenous ligands engage TLRs in order to make use of the innate immune machinery to mount an infection-independent immune response. Interfering with the engagement of TLRs activating CNS-resident cells poses a potential therapeutic strategy for the treatment of sterile neuroimmune disorders such as MS.

\section{Methods}

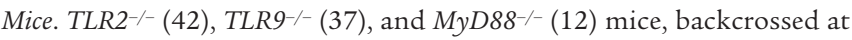
least 8 times, were kindly provided by S. Akira and K. Takeda (Osaka University, Osaka, Japan) and H. Wagner (Technical University of Munich). CD45 congenic C57BL/6 Ly5.1 mice (CD45.1) were purchased from the Jackson Laboratory, and all mice were kept and bred in-house under pathogen-free conditions.

Generation of BM chimeric mice. BM chimeric mice were generated as described recently $(23,24,26)$. In brief, 6 - to 8 -week-old recipient mice were reconstituted with $\mathrm{BM}$ cells derived from tibiae and femurs from the respective donors. $\mathrm{BM}$ cells $\left(5 \times 10^{6}\right.$ cells $)$ were injected into the tail veins of recipients conditioned by whole-body irradiation (1100 cGy) 24 hours earlier. From 6 to 8 weeks after grafting, reconstitution was assessed by FACS analysis of peripheral blood. Blood samples were prepared at $4{ }^{\circ} \mathrm{C}$ in buffer solution (PBS containing $2 \%$ FCS and $0.2 \%$ NaN3) and stained with FITC-labeled CD45.2 (BD Biosciences - Pharmingen) and PElabeled CD45.1 antibody (BD Biosciences - Pharmingen). After lysis of erythrocytes with FACS lysis solution (BD) and washing, cell suspensions were analyzed on a FACSCalibur (BD). Data were acquired with CellQuest software version $3.3(\mathrm{BD})$.

Induction of EAE. Female 6- to 10-week-old mice (12- to 16-week-old BM chimeric mice, respectively) of each group were immunized subcutaneously with $200 \mu \mathrm{g}$ of MOG $_{35-55}$ peptide emulsified in CFA containing $500 \mu \mathrm{g}$ of M. tuberculosis (H37RA, Difco; BD Diagnostics). The mice received intraperitonal injections with $250 \mathrm{ng}$ PTX (Sigma-Aldrich) at the time of immunization and 48 hours later. After 7 days, the mice received an identical booster with MOG/CFA without PTX. For adoptive transfer, donor mice were immunized subcutaneously with $200 \mu \mathrm{g} \mathrm{MOG}_{35-55}$ in CFA containing $500 \mu \mathrm{g}$ of M. tuberculosis. Immediately after and 2 days later, mice received 250 ng PTX. At 11 dpi, mice were killed, spleens were removed and homogenized, and RBCs were lysed. Cells were cultured for 4 days in RPMI1640 medium supplemented with $10 \% \mathrm{FCS}, 10 \mathrm{mg} / \mathrm{ml}$ MOG peptide, and 2.5 $\mathrm{ng} / \mathrm{ml}$ murine recombinant IL-12 (R\&D Systems), then harvested; dead cells were removed by Ficoll (Biochrom AG) centrifugation. Cells were then injected into recipient mice $\left(25 \times 10^{6}\right.$ cells/mouse $)$. Each animal received 250 ng PTX on days 0 and 2 after transfer. The animal care and use committee of the University of Göttingen approved all experiments.

Clinical evaluation. The mice were scored daily as follows: 0 , no detectable signs of EAE; 0.5 , distal section of tail paralyzed; 1.0, distal and proximal sections of tail paralyzed; 1.5 , distal and proximal sections of tail paralyzed and hind-limb weakness; 2 , unilateral partial hind-limb paralysis; 2.5 , bilateral partial hind-limb paralysis; 3, complete bilateral hind-limb paralysis; 3.5, complete hind-limb paralysis and unilateral forelimb paralysis; 4 , total paralysis of forelimb and hind limb; and 5, death. Statistical analysis was performed using an unpaired Student's $t$ test, and $P$ values less than or equal to 0.05 were considered significant. 
Histology. Mice were euthanized with $\mathrm{CO}_{2}$. Spinal cords were removed and fixed in $4 \%$ buffered formalin. Later, spinal cords were dissected and paraffin embedded before staining with $\mathrm{H} \& \mathrm{E}$, luxol fast blue to assess the degree of demyelination, Mac-3 (BD Biosciences - Pharmingen) for macrophages, CD3 for T cells (Serotec), and APP (Chemicon International).

Flow cytometry and in vivo depletion of Treg cells. For flow cytometry, cells were stained with primary Abs (CD45.1, CD45.2, CD11b, CD4, CD25, and CD45RB; all from BD Biosciences - Pharmingen) for 30 minutes at $4{ }^{\circ} \mathrm{C}$. The cells were washed and analyzed using FACSCalibur (BD). Viable cells were gated by forward and side scatter of light. Data were acquired with CellQuest software version 3.3 (BD). Postacquisition analysis was performed using WinMDI 2.8 software (The Scripps Research Institute). In vivo depletion of $\mathrm{CD}^{+} \mathrm{CD} 25^{+} \mathrm{T}$ cells was achieved by intravenous injection of $100 \mu \mathrm{g}$ of monoclonal anti-CD25 antibody (clone PC61). Control mice were injected with rat Ig (all from BD Biosciences - Pharmingen). Depletion of Treg cells was confirmed by staining peripheral blood lymphocytes for CD 4 and CD25 markers on day 3 after injection of the anti-CD25 antibody.

Recall responses. Mice were primed by flank injections of MOG/CFA. After $7 \mathrm{dpi}$, the draining axillary and inguinal LNs were removed and homogenized. LN cells $\left(5 \times 10^{5}\right)$ were placed as triplicates in 96-well plates and pulsed with $50 \mu \mathrm{g} / \mathrm{ml}$ MOG peptide or KLH (Sigma-Aldrich) as control. Cultures were pulsed with $0.5 \mu \mathrm{Ci}$ of $\left[{ }^{3} \mathrm{H}\right]$ thymidine per well $(1 \mathrm{mCi} / \mathrm{ml}$ ${ }^{3} \mathrm{HTdR}$; Amersham Pharmacia Biotech) during the last 18 hours of 2 days culture of $T$ cells. Cells were harvested onto glass fiber filter membranes by using a 96-well plate cell harvester (IH110-96, Inotech AG), and tritiated thymidine incorporation was measured by scintillation counting (MicroBeta Plus 1450 reader; PerkinElmer). For cytokine analysis, sister cultures were harvested 4 hours after culture supernatants were analyzed by ELISA for IFN- $\gamma$ (R\&D Systems).

Real-time PCR and luciferase assay. RNA was extracted from acutely diseased spinal cords of mice with EAE. The spinal cord was flushed with ice-cold HBSS, and RNA was isolated using RNAeasy Mini Kits (QIAGEN) following the manufacturer's instructions. The samples were treated with DNaseI (Roche Diagnostics Corp.), and $1 \mu \mathrm{g}$ of RNA was transcribed into cDNA using oligo-dT primers and the SuperScript II RT kit (Invitrogen Corp.). cDNA (2.5 $\mu \mathrm{l})$ was transferred into a 96-well Multiply PCR-plate (SARSTEDT), and $12.5 \mu \mathrm{l}$ ABsolute QPCR SYBER Green Master Mix

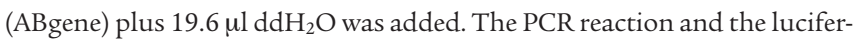
ase assay were performed as described recently (43). The following primer probe pairs were used: TLR1, sense CAAACGCAAACCTTACCAGAGTG, antisense GAGATTCGGGGTCTTCTTTTTCC; TLR2, sense AAAATGTCGTTCAAGGAG, antisense TTGCTGAAGAGGACTGTT; TLR3, sense ACTTGCTATCTTGGATGC, antisense AGTTCTTCACTTCGCAAC;
TLR4, sense TCCCTGATGACATTCCTTCTT, antisense TGAGCCACATTGAGTTTCTTTA; TLR5, sense CGCCTCCATTCTTCATTCCG, antisense CCTTCAGTGTCCCAAACAGTCG; TLR6, sense CTTACTCGGAGACAGCACTGAAGTC, antisense GCAGGTGGGTGACATCTTTAGG; TLR7, sense TGACTCTCTTCTCCTCCA, antisense GCTTCCAGGTCTAATCTG; TLR8, sense CTGTCCAAGGTGTTACAATGCTCC, antisense TTGAGAGAGGTTTCCGAAGACG; TLR9, sense GGTGTGGAACATCATTCT, antisense ATACGGTTGGAGATCAAG; MyD88, sense CTGGGTGAAGAAGCTACTCCAT, antisense TAGAAACTTGGGAGGTGTGTGG; and GAPDH, sense TCCTGCACCACCAACTGCTTAGCC, antisense GTTCAGCTCTGGGATGACCTTGCC.

Statistics. Statistical differences of clinical scores were evaluated using a 2-tailed unpaired Student's $t$ test. Differences were considered significant when the $P$ value was less than 0.05 . ELISA and proliferation assays are expressed as average of triplicate wells \pm SEM.

\section{Acknowledgments}

We thank S. Ebert, O. Dell, and S. Fichte for excellent technical assistance. We are grateful to R. Nau, (University of Göttingen, Göttingen, Germany) and M. Heikenwälder (University Hospital Zurich, Zurich, Switzerland), for helpful scientific discussion and to $\mathrm{H}$. Weighardt (Institute of Medical Microbiology) for help with mouse delivery. Assistance through grants was provided by Fritz Thyssen Stiftung and the Gemeinnützige Hertie-Stiftung (GHST) (to M. Prinz) and by the National Center for Excellence in Research (NCCR) in Switzerland and the GHST (to B. Becher). H. Schmidt is a fellow of the Gertrude Reemtsma Foundation. I. Gutcher is a Meyerhof Foundation fellow, and B. Becher is a Harry Weaver Neuroscience scholar of the National MS Society, New York, New York, USA.

Received for publication June 27, 2005, and accepted in revised form November 22, 2005.

Address correspondence to: Marco Prinz, Institute of Neuropathology, Georg-August-University, Robert-Koch-Strasse 40, D-37075 Gottingen, Germany. Phone: 49-551-39-14340; Fax: 49-551-39-8472; E-mail: mprinz@med.uni-goettingen.de. Or to: Burkhard Becher, Neurology Clinic, Division of Neuroimmunology, University Hospital Zurich, Frauenklinikstrasse 10, 8091 Zurich, Switzerland. Phone: 41-1-255-8842 or 41-1-255-5500; Fax: 411-255-9765; E-mail: burkhard.becher@usz.ch.

Marco Prinz and Folker Garbe contributed equally to this work.
1. Steinman, L. 1996. Multiple sclerosis: a coordinated immunological attack against myelin in the central nervous system. Cell. 85:299-302.

2. Owens, T., Wekerle, H., and Antel, J. 2001. Genetic models for CNS inflammation. Nat. Med. 7:161-166.

3. Banchereau, J., and Steinman, R.M. 1998. Dendritic cells and the control of immunity. Nature. 392:245-252.

4. Schnare, M., et al. 2001. Toll-like receptors control activation of adaptive immune responses. Nat. Immunol. 2:947-950.

5. Takeda, K., and Akira, S. 2005. Toll-like receptors in innate immunity. Int. Immunol. 17:1-14.

6. Beutler, B. 2004. Inferences, questions and possibilities in Toll-like receptor signalling. Nature. 430:257-263.

7. Matzinger, P. 1994. Tolerance, danger, and the extended family. Annu. Rev. Immunol. 12:991-1045.

8. Lemaitre, B., Nicolas, E., Michaut, L., Reichhart, J.M., and Hoffmann, J.A. 1996. The dorsoventral regulatory gene cassette spatzle/Toll/cactus controls the potent antifungal response in Drosophila adults. Cell. 86:973-983.

9. Medzhitov, R., et al. 1998. MyD88 is an adaptor protein in the hToll/IL-1 receptor family signaling pathways. Mol. Cell. 2:253-258.

10. Muzio, M., Ni, J., Feng, P., and Dixit, V.M. 1997. IRAK (Pelle) family member IRAK-2 and MyD88 as proximal mediators of IL-1 signaling. Science. 278:1612-1615.

11. Prinz, M., et al. 2003. Prion pathogenesis in the absence of Toll-like receptor signalling. EMBO Rep. 4:195-199.

12. Adachi, O., et al. 1998. Targeted disruption of the MyD88 gene results in loss of IL-1- and IL-18-mediated function. Immunity. 9:143-150.

13. Kawai, T., Adachi, O., Ogawa, T., Takeda, K., and Akira, S. 1999. Unresponsiveness of MyD88-deficient mice to endotoxin. Immunity. 11:115-122.

14. Takeuchi, O., et al. 2000. Cellular responses to bac- terial cell wall components are mediated through MyD88dependent signaling cascades. Int. Immunol. 12:113-117.

15. Hemmi, H., et al. 2002. Small anti-viral compounds activate immune cells via the TLR7 MyD88-dependent signaling pathway. Nat. Immunol. 3:196-200.

16. Hacker, H., et al. 2000. Immune cell activation by bacterial CPG-DNA through myeloid differentiation marker 88 and tumor necrosis factor receptor-associated factor (TRAF)6. J. Exp. Med. 192:595-600.

17. Hayashi, F., et al. 2001. The innate immune response to bacterial flagellin is mediated by Tolllike receptor 5. Nature. 410:1099-1103.

18. Segal, B.M., Chang, J.T., and Shevach, E.M. 2000. $\mathrm{CPG}$ oligonucleotides are potent adjuvants for the activation of autoreactive encephalitogenic T cells in vivo. J. Immunol. 164:5683-5688.

19. Olson, J.K., and Miller, S.D. 2004. Microglia initiate central nervous system innate and adap- 
tive immune responses through multiple TLRs. J. Immunol. 173:3916-3924.

20. Lehnardt, S., et al. 2003. Activation of innate immunity in the CNS triggers neurodegeneration through a Toll-like receptor 4-dependent pathway. Proc. Natl. Acad. Sci. U. S. A. 100:8514-8519.

21. Bsibsi, M., Ravid, R., Gveric, D., and van Noort, J.M. 2002. Broad expression of Toll-like receptors in the human central nervous system. J. Neuropathol. Exp. Neurol. 61:1013-1021.

22. Pasare, C., and Medzhitov, R. 2004. Toll-dependent control mechanisms of CD4 T cell activation. Immunity. 21:733-741.

23. Becher, B., Durell, B.G., Miga, A.V., Hickey, W.F., and Noelle, R.J. 2001. The clinical course of experimental autoimmune encephalomyelitis and inflammation is controlled by the expression of CD40 within the central nervous system. J. Exp. Med. 193:967-974.

24. Becher, B., Durell, B.G., and Noelle, R.J. 2003. IL-23 produced by CNS-resident cells controls T cell encephalitogenicity during the effector phase of experimental autoimmune encephalomyelitis. J. Clin. Invest. 112:1186-1191. doi:10.1172/ JCI200319079.

25. Greter, M., et al. 2005. Dendritic cells permit immune invasion of the CNS in an animal model of multiple sclerosis. Nat. Med. 11:328-334.

26. Prinz, M., et al. 2003. Positioning of follicular dendritic cells within the spleen controls prion neuro- invasion. Nature 425.957-962.

27. Asheuer, M., et al. 2004. Human CD34+ cells differentiate into microglia and express recombinant therapeutic protein. Proc. Natl. Acad. Sci. U. S. A. 101:3557-3562.

28. Hickey, W.F., Vass, K., and Lassmann, H. 1992. Bone marrow-derived elements in the central nervous system: an immunohistochemical and ultrastructural survey of rat chimeras. J. Neuropathol. Exp. Neurol. 51:246-256.

29. Priller, J., et al. 2001. Targeting gene-modified hematopoietic cells to the central nervous system: use of green fluorescent protein uncovers microglial engraftment. Nat. Med. 7:1356-1361.

30. Compston, A., and Coles, A. 2002. Multiple sclerosis. Lancet. 359:1221-1231.

31. Casetta, I., and Granieri, E. 2000. Prognosis of multiple sclerosis:environmental factors. Neurol. Sci. 21:S839-S842.

32. Buljevac, D., et al. 2002. Prospective study on the relationship between infections and multiple sclerosis exacerbations. Brain. 125:952-960.

33. Oksenberg, J.R., et al. 1993. Selection for T-cell receptor $\mathrm{V} \beta-\mathrm{D} \beta$-J $\beta$ gene rearrangements with specificity for a myelin basic protein peptide in brain lesions of multiple sclerosis. Nature. 362:68-70.

34. Heppner, F.L., et al. 2005. Experimental autoimmune encephalomyelitis repressed by microglial paralysis. Nat. Med. 11:146-152.

35. Quesniaux, V., et al. 2004. Toll-like receptor path- ways in the immune responses to mycobacteria. Microbes Infect. 6:946-959.

36. Pasare, C., and Medzhitov, R. 2003. Toll pathway-dependent blockade of CD4+CD25+ T cellmediated suppression by dendritic cells. Science. 299:1033-1036.

37. Hemmi, H., et al. 2000. A Toll-like receptor recognizes bacterial DNA. Nature. 408:740-745.

38. Yasuda, K., et al. 2005. Endosomal translocation of vertebrate DNA activates dendritic cells via TLR9dependent and -independent pathways. J. Immunol. 174:6129-6136.

39. Bauer, J., et al. 1998. T-cell apoptosis in inflammatory brain lesions. Destruction of T cells does not depend on antigen recognition. Am. J. Pathol. 153:715-724.

40. Leadbetter, E.A., et al. 2002. Chromatin-IgG complexes activate B cells by dual engagement of IgM and Toll-like receptors. Nature. 416:603-607.

41. Kerfoot, S.M., et al. 2004. TLR4 contributes to disease-inducing mechanisms resulting in central nervous system autoimmune disease. J. Immunol. 173:7070-7077.

42. Werts, C., et al. 2001. Leptospiral lipopolysaccharide activates cells through a TLR2-dependent mechanism. Nat. Immunol. 2:346-352.

43. Koedel, U., et al. 2003. Toll-like receptor 2 participates in mediation of immune response in experimental pneumococcal meningitis. J. Immunol. 170:438-444. 